\title{
Intelligent and Scalable Air Quality Monitoring with 5G Edge
}

Su, Xiang

2021

Su , X, Liu , X, Hossein Motlagh , N , Cao , J , Su, P, Pellikka , P, Liu , Y, Petäjä , T , Kulmala , M , Hui , P \& Tarkoma , S 2021 , ' Intelligent and Scalable Air Quality Monitoring with 5G Edge ' , IEEE Internet Computing , vol. 25 , no. 2 , pp. 35-43 . https://doi.org/10.1109/MIC.2021.3059189

http://hdl.handle.net/10138/331666

https://doi.org/10.1109/MIC.2021.3059189

acceptedVersion

Downloaded from Helda, University of Helsinki institutional repository.

This is an electronic reprint of the original article.

This reprint may differ from the original in pagination and typographic detail.

Please cite the original version. 


\title{
Intelligent and Scalable Air Quality Monitoring with $5 \mathrm{G}$ Edge
}

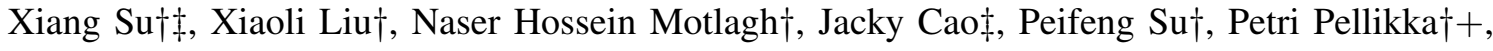 \\ Yongchun Liu*, Tuukka Petäjä†, Markku Kulmala†, Pan Hui†§, Sasu Tarkoma† \\ $\dagger$ University of Helsinki, Helsinki, Finland \\ $\ddagger$ University of Oulu, Oulu, Finland \\ +Wuhan University, Wuhan, China \\ *Beijing University of Chemical Technology, Beijing, China \\ $\S$ The Hong Kong University of Science and Technology, Hong Kong
}

\begin{abstract}
Air pollution introduces a major challenge for societies, where it leads to the premature deaths of millions of people each year globally. Massive deployment of air quality sensing devices and data analysis for the resultant data will pave the way for the development of real-time intelligent applications and services, e.g., minimization of exposure to poor air quality either on an individual or city scale. 5G and edge computing supports dense deployments of sensors at high resolution with ubiquitous connectivity, high bandwidth, high-speed gigabit connections, and ultra-low latency analysis. This paper conceptualizes AI-powered scalable air quality monitoring and presents two systems of calibrating low-cost air quality sensors and the image processing of pictures captured by hyperspectral cameras to better detect air quality. We develop and deploy different AI algorithms in these two systems on a 5G edge testbed and perform a detailed analytics regarding to 1) the performance of $\mathrm{AI}$ algorithms and 2) the required communication and computation resources.
\end{abstract}

Index Terms-5G, edge computing, air quality monitoring, sensor calibration, hyperspectral images processing.

\section{INTRODUCTION}

In recent years, we have witnessed an unprecedented growth of urban areas, which are characterized by highly dense and mobile sensing devices, localized data processing, and versatile actuators. It has paved the way for novel, personalized, real-time, clean, and safe digital city services for citizens. To enable this, future smart cities provide intelligent and green living environments at lower costs, and are supported by Artificial Intelligence (AI) and 5G. Numerous intelligent nodes, such as base stations, road side units, and even smart street lights provide near real-time decision making and optimization [1].

Air quality degradation in urban areas has become a global challenge for human health, ecosystems, and the climate. The recent study by the Global Burden of Disease project has reported that 5.5 million people worldwide are dying prematurely each year as a result of low-quality air. [2]. Air pollutants are conventionally measured by expensive highend stationary stations. However, high costs and the need for frequent station maintenance prevents dense and large-scale deployments. Recent advances in sensing technologies and wireless communications enable a complementary approach of large scale sensing solutions with low-cost sensing devices [3].
We address the significant problem of real-time air quality sensing with high spatial resolutions by leveraging $5 \mathrm{G}$ edge computing, AI methods, and dense deployment of low-cost sensors and hyperspectral cameras. For massive deployment of low-cost sensors, we focus on calibrating a large number of them with a small number of highly accurate reference stations by using AI techniques for improved air quality measurements. For the hyperspectral cameras, we infer the air quality indicators based on captured spectral images through a Convolutional Neural Network (CNN).

$5 \mathrm{G}$ edge computing offers unification through supporting versatile connections and a framework for managing smart city deployment. 5G with low latency and high bandwidth carries the data needed by AI algorithms for real-time analysis. Edge servers, typically co-located with $5 \mathrm{G}$ base stations, allow for AI algorithms to be deployed in proximity to sensing devices and end users. By allowing resource-constrained devices (lowcost sensors and hyperspectral cameras) to offload data and computation-intensive tasks to edge servers, we enable lowlatency air quality analysis, preserve computation capacities; conserve device battery life; and support scalable, secure, and privacy-preserving services.

We propose AI-powered scalable real-time air pollution monitoring by developing a sensing architecture with different air quality sensing devices, from resource-constrained immobile and mobile sensing devices to cameras (as depicted in Figure 11. We demonstrate the feasibility in deploying AI algorithms, i.e., calibration and image processing, on a realworld 5G Test Network (5GTN) [4] and conduct a comprehensive scalability analytics of system latency, throughput and computation resources on edge servers.

\section{Scalable Air Quality Monitoring With 5G Edge}

\section{A. Challenges}

High resolution spatio-temporal air quality monitoring requires dense and massive-scale low-cost sensor and hyperspectral camera deployment in urban areas. This introduces significant challenges, including 1) ubiquitous connectivity and high-speed connections; 2) the processing of big data volumes; and 3) real-time analysis for latency sensitive applications. In 


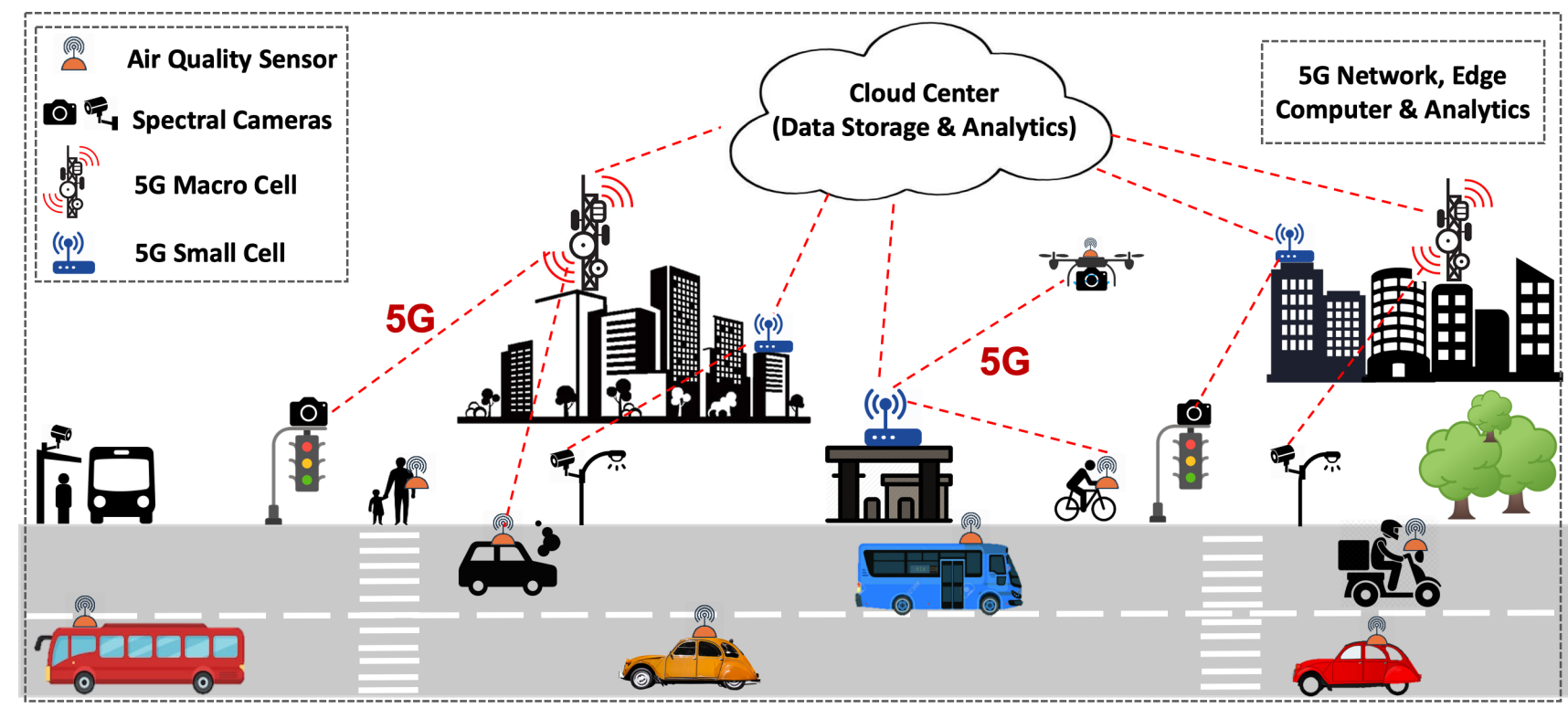

Fig. 1: A conceptual 5G edge architecture for massively deploying air quality sensors and spectral cameras in different locations within urban environments. Ubiquitous fixed and mobile air quality devices generate large volumes of data requiring reliable and high-speed data connections to edge computers (co-located with 5G small cells) at their proximity for fast data analysis. Measured data can be sent to the cloud centers for further long-term analysis and storage.

the scenario of a single city block, there would be thousands of air quality sensors deployed while each producing kilobytes of air quality data per measurement, as well as hundreds of hyperspectral cameras with each generating images of 30-300 megabytes in about a minute. Both types of devices deliver large volumes of air quality data at high speed, which requires real-time AI analysis. The following are crucial challenges:

1) Ubiquitous connectivity Heterogeneous and multiplatform sensing devices are distributed in urban environments and underpin air quality sensing infrastructure and services. Novel communication technologies are required to provide connectivity to everything at everywhere and every time. This allows devices to create, share, and process data in the air quality sensing infrastructure.

2) High-speed gigabit connections Diverse systems expect high-speed connections to carry data needed by AI algorithms to perform real-time analysis, reasoning, and optimizations. For example, high-speed connections enable faster air quality detection with hyperspectral images.

3) Big data streams processing capacities The amount of data streams collected from air quality devices is enormous and data is continuously generated at high frequencies. Potential subsequent integration of multiple sources further amplifies this challenge. Processing big air quality data streams extracts higher level information, guides the understanding of complex environmental situations, and enables real-time analysis to provide insights for users.

4) Low-latency analysis For latency sensitive services, the ultimate goal is to generate timely insights of environ- mental situations before becoming obsolete. For example, city navigation would benefit considerably from real-time air quality sensing, as this could inform users whether particular routes are suitable for their own personal safety levels and they can avoid unnecessary exposure to harmful particulates (e.g., air pollution, aerosols that may transport infectious viruses). Air quality monitoring demands high processing throughput, which introduces a conflict of latency requirement.

\section{B. $5 G$ edge based air quality sensing}

As shown in Figure 1] we propose the 5G edge to address these challenges. Massive scale sensor deployment generates big data streams, which requires bandwidths of up to tens or hundreds of gigabytes per second per city block from the communication network. Current 4G networks only support limited number of connections, and Narrow-Band IoT (NBIoT) cannot provide sufficient bandwidth to support transmission of large data volumes. 5G networks allow for both massive numbers of connections and provide support for the transmission of large amounts of data.

5G networks are mobile networks supporting densely deployed massive-scale air quality sensors in urban areas horizontally and vertically. The dense deployment of $5 \mathrm{G}$ base stations, equipped with antenna arrays, significantly increases the available line-of-sights between sensing devices and 5G antennas. This allows for the estimation of the precise 3D locations of sensors and therefore offers accurate air quality information. 


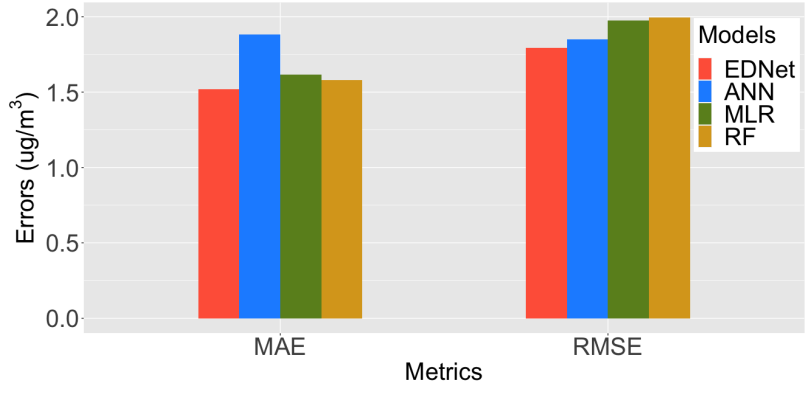

(a)

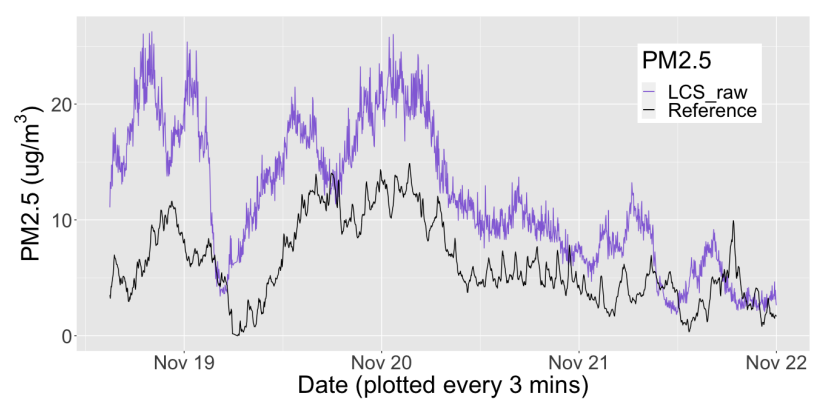

(c)

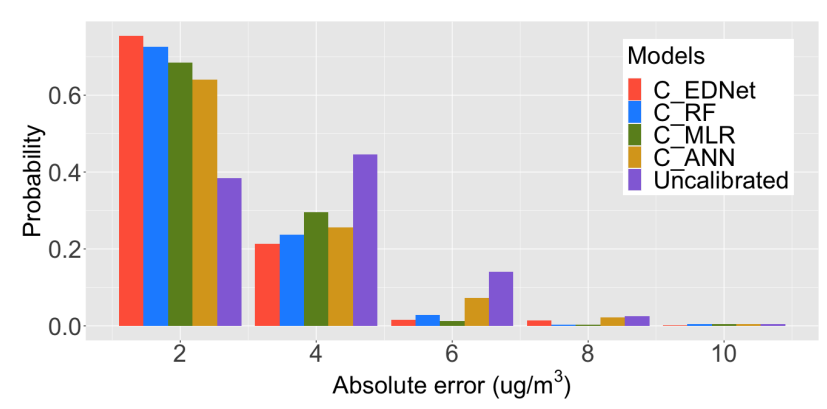

(b)

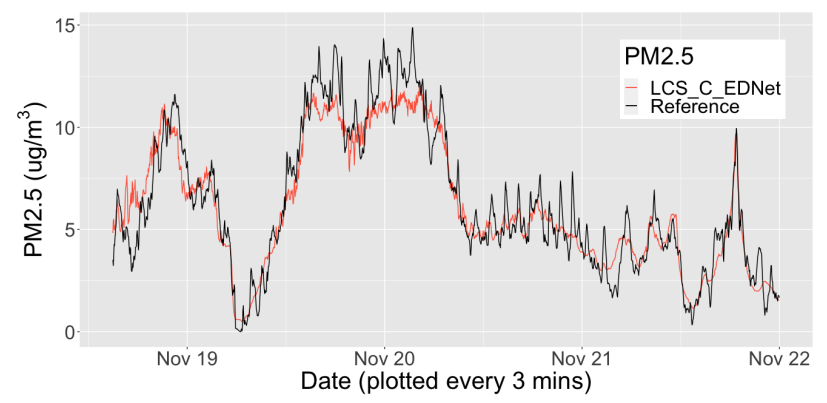

(d)

Fig. 2: (a) RMSE and MAE between true values of reference station and predictions for different calibration models. (b) Absolute errors distributions of different calibration models and absolute error distribution of measurements from low-cost sensors. Calibration decreases the errors dramatically by deploying the calibration models. Raw data from low-cost sensors (uncalibrated) has the lowest percentage for absolute errors within interval $[0,2]$ and has the highest percentage for absolute errors within interval [2,4], [4,6], and [6,8]. (c) Raw data of $\mathrm{PM}_{2.5}$ from low-cost sensor compared with true $\mathrm{PM}_{2.5}$ values from reference station. (d) Calibrated $\mathrm{PM}_{2.5}$ of low-cost sensor by EDNet compared with true $\mathrm{PM}_{2.5}$ values from reference station. It shows that EDNet is able to recover the real $\mathrm{PM}_{2.5}$ values to a certain extent.

5G networks with their massive Machine Type Communications (mMTC) characteristic fulfill the requirement of ultradense machine communications by supporting connection densities of one million devices per square kilometer and fulfilling certain quality of service requirements. Thus, mMTC enables the connection of thousands of air quality sensors and hyperspectral cameras simultaneously. The enhanced Mobile Broadband (eMBB) 5G feature supports high data rates (exceeding $10 \mathrm{Gbps}$ ), which fulfills the throughput requirement, when thousands of air quality sensors and cameras transmits large volumes of data simultaneously and may require gigabytes of network capacity. Finally, 5G offers ultra-reliable and Lowlatency Communications (uRLLC), which fulfills the requirements for extremely low latency communication, allowing for very fast raw data transmission as well as delivering processed air quality information in real-time to citizens [5].

Edge server offers data storage and analysis at the edge of networks, which enables reduced network traffic, low and predictable latency, secure and privacy-preserving services and applications, and scalability [6]. To support the storage and processing of large volumes of air quality data collected from different locations within a city block, edge servers enable location-based real-time air quality analysis. Here, we focus on implementing AI algorithms, i.e., sensor data calibrations and image processing on edge servers which are in proximity to sensing devices.

\section{DATA ANALYSIS FOR AIR QUALITY SENSING ON THE EDGE}

This section presents two AI systems for air quality monitoring, i.e., air quality sensing with calibration methods and hyperspectral imaging with deep learning methods, both deployable on the $5 \mathrm{G}$ edge.

\section{A. Calibration for low-cost air quality sensors}

Accurate air quality sensing in cities requires periodic calibration of massive low-cost sensors in near real-time, because low-cost sensors suffer from accuracy, instability, and sensor drifts. Advances in air quality sensing have led to AI-based calibration methods for enhancing sensor accuracy [7]. Calibration is traditionally performed by locating low-cost sensors near reference sensing stations. Massive calibration of air quality sensors generates significant amounts of data. This data is sent and received from reference stations and low-cost sensors to edge computers, which requires fast data communication and calibration within the network.

We evaluate the calibration performance of air quality sensors equipped with $\mathrm{WiFi}$, with using a smartphone as a 
WiFi hotspot. The sensor unit is capable of sensing and reporting timestamped data about particulate mass concentration (PM), gas pollutants, such as carbon monoxide and ozone, meteorological parameters, such as temperature and humidity, and instrument parameters, such as device battery level. PM with diameters less than 2.5 microns $\left(\mathrm{PM}_{2.5}\right)$ are measured using Sensirion SPS30 with a red laser arranged in a 90 degree scattering angle to observe the sample airflow generated by a fan [8].

We calibrate the readings of $\mathrm{PM}_{2.5}$ using measurements from low-cost sensors, as $\mathrm{PM}_{2.5}$ is one of major air pollutants affecting everyone's lives. To perform calibration, we collected an accurate dataset containing $\mathrm{PM}_{2.5}$ for continuous 45 days in 2019 from the air quality reference station in Helsinki called SMEAR-III [9]. $\mathrm{PM}_{2.5}$ in the reference station is measured using a TEOM 1405-D sensor. We also collected a dataset containing the measurements of $\mathrm{PM}_{2.5}$, pressure, temperature, and relative humidity using low-cost sensors while installed near the reference station within the same period.

Regression models, including multiple linear regression (MLR), random forest (RF), and artificial neural network (ANN), have been widely used in sensor calibration for air quality monitoring [10]. Such models use current time sensor measurements as inputs without taking the temporal structure into consideration. Long short-term memory (LSTM) is designed for incorporating temporal structure and is well suited for modeling time series. We propose EDNet, an EncoderDecoder LSTM architecture for sensor calibration for air quality monitoring. The architecture includes an LSTM encoder and an LSTM decoder, which are responsible for extracting the observation information of input values and obtaining the features by feeding the input sequences into an LSTM, and reading the encoded input sequence and making predictions for each element in the output sequence, respectively. Fully connected dense layers are used to process the time steps produced by the decoder to produce the final outputs. The former $80 \%$ of collected data is used for training and the latter $20 \%$ is used for testing. In the training set, the latter $30 \%$ of data is used for validation. PM measurements along with environmental factors collected from low-cost sensors are used for building the calibration model, and two hours window of measurements are used as input sequences.

The baselines we consider are an MLR model, a RF predictor, and an ANN based method denoted by ANN, which consists of two hidden layers with 64 and 32 neurons, respectively. Figure 2(a) presents the root mean squared error (RMSE) and mean absolute error (MAE) of EDNet compared to baselines. RF suitable for capturing nonlinear relationships has comparable performance to MLR. ANN has better performance compared to RF and MLR from the RMSE perspective. While EDNet presents the best performance from RMSE and MAE perspectives, because it takes the temporal information of measurements from different sensors into consideration. Calibration decreases the errors dramatically by deploying calibration models (Figure 2(b)]. Figures 2(c) and 2(d) present the real-time calibration performance of EDNet for four days.

\section{B. Hyperspectral Image Processing}

Massive usage of hyperspectral cameras is foreseen to be an integral part of future sensing solutions for air quality monitoring [11], as hyperspectral cameras collect detailed information from images for air quality detection. Dark channel prior [12] is a widely used prior for image dehazing, and from the prior, a hyperspectral image should obtain a more accurate dark channel than RGB images as there are many more colors in a hyperspectral image. Different wavelengths have different responses to haze, indicating that more information should be obtained from a hyperspectral image than a related RGB image [13]. When applying a hyperspectral camera for air quality sensing, air pollution changes the path of light, causing captured images under inclement weather to have low contrast and limited visibility [14]. We utilize a portable handheld push broom hyperspectral camera, called Specim IQ [15]. The camera covers a spectral range from 400 to $1000 \mathrm{~nm}$, and the resolution of a captured hyperspectral image is $512 \times 512 \times 204$. We select 51 channels out from the 204 channels for the retrieval of atmospheric visibility.

To process hyperspectral images for air pollution detection, we develop a model, i.e., EnvNet, to evaluate the relationship between images and atmospheric visibility. EnvNet consists of several convolutional layers and ResNet blocks [16], one pooling layer, and one fully connected layer. EnvNet gives a predicted atmospheric visibility value for each image, which can be compared with the reference value from the instrument. We collect 1487 hyperspectral images using the Specim IQ camera at Beijing University of Chemical Technology (BUCT). Two image samples are shown in Figure 3 representing different visibility. We use $80 \%$ of the data for training and $20 \%$ for testing. For the training set, $20 \%$ of the data is used for validation. The reference atmospheric visibility values are provided by the instrument PWD22, Vaisala of BUCT [17].

For atmospheric visibility, MAE and the coefficient of determination $\left(R^{2}\right)$ are used to evaluate model performance. The MAEs between the predicted values and reference values for EnvNet, AlexNet [18], and DenseNet [19] are 698.65 meters, 937.46 meters, and 1172.05 meters, respectively (Figure 3(c)). The $R^{2} \mathrm{~s}$ between the predicted values and reference values for EnvNet, AlexNet, and DenseNet are 0.95, 0.94, and 0.92, respectively. EnvNet outperforms AlexNet and DenseNet by achieving the highest $R^{2}$ and the lowest MAE.

\section{EXPERIMENTATION AND ANALYTICS}

\section{A. $5 G$ Edge Testbed}

Our experiments demonstrate the feasibility and performance of deploying the above-mentioned AI systems and algorithms on a real-world $5 \mathrm{G}$ edge testbed for massive-scale air quality monitoring. Our testbed is composed of 5GTN, edge servers, as well as air quality sensors and hyperspectral cameras. $5 \mathrm{GTN}$ is a full scale $5 \mathrm{G}$ micro operator and the world's first open $5 \mathrm{G}$ test network. 5GTN provides non-standalone $5 \mathrm{G}$ and LTE connectivity through deploying different $5 \mathrm{G}$ 


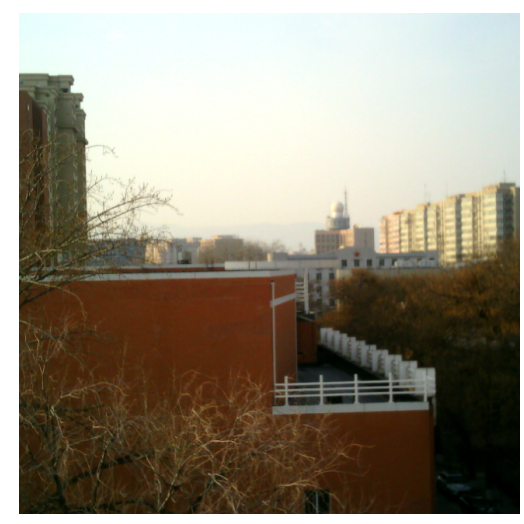

(a)

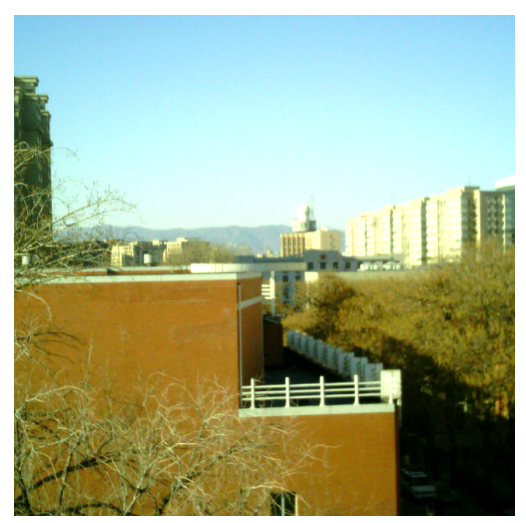

(b)

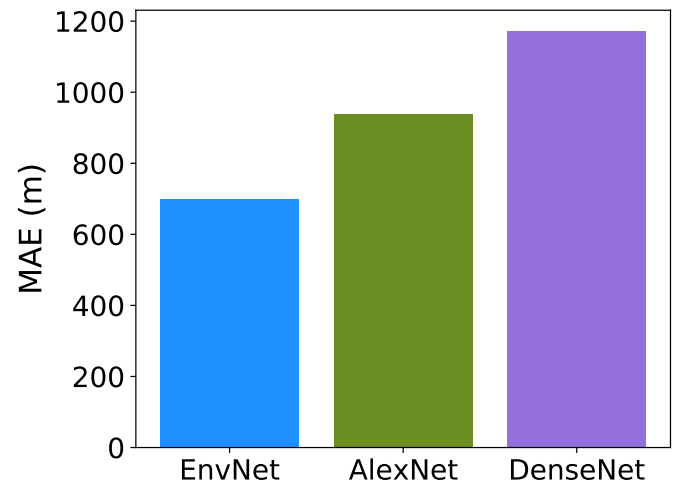

(c)

Fig. 3: (a) and (b) Two images captured on two different days with different atmospheric visibility. The range for visibility is from 0 to $20 \mathrm{~km}$, where $0 \mathrm{~km}$ means that the air is heavily polluted and $20 \mathrm{~km}$ refers to a relatively clear day. (c) Mean absolute errors of EnvNet, AlexNet, and DenseNet.

and LTE base stations and is controlled by operator-grade evolved packet cores, which provides key functions, such as mobility management, data packet routing, and authentication. We conduct our experimentation at the University of Oulu implementation of $5 \mathrm{GTN}$, which has air interfaces of two $5 \mathrm{G}$ macro cells (n78), several LTE macro cells (B28, B7, B42), and a LoRa network supporting frequencies [0.7, 2.1, 2.6 and 3.5] GHz. Moreover, testbed supports heterogeneous wireless technologies, including IEEE 802.11, Bluetooth LE, LoRa, NB-IoT, UWB and LTE evolutions, such as LTE-M and LTEU. Edge servers are deployed on 5GTN to support latencysensitive data analysis. The edge server in this experimentation has an Intel Core i7-8700 CPU, 32 GB memory, and an NVIDIA GeForce RTX 2080 Ti GPU.

A PC (Intel Core i7-8700K CPU, 32 GB memory, and an NVIDIA GeForce RTX 2080 with Max-Q Design GPU) relays the real collected data from low-cost air quality sensors and hyperspectral cameras. With this approach, we are able to evaluate massive deployment of devices through multiple parallel threads (one thread represents one device). Data is sent from each thread to a OnePlus 7 Pro 5G smartphone through local $5 \mathrm{GHz} 802.11 \mathrm{ac}$ WiFi connections. The smartphone then forwards the data using either $5 \mathrm{G}$ or LTE connections to an edge server, which is connected to $5 \mathrm{GTN}$ through a $5 \mathrm{G}$ modem.

\section{B. Results and analytics}

To analyze the performance of $5 \mathrm{G}$ edge for scalable air quality monitoring, we study:

- Data transmission latency and throughput. We separate overall latency into latency of transmitting data from devices to a smartphone with WiFi, and latency of forwarding the data from the smartphone to an edge server with $5 \mathrm{G}$ or LTE.

- Scalability. An increasing amount of devices are emulated to deliver data to an edge server.
- Required computation resources (CPU, GPU, and memory) of two systems with alternative AI algorithms on edge servers.

We analyze communication latency, throughput, and required computation resources on edge servers with an incremental number of devices (by increasing number of threads). We consider future dense deployment of devices in ubiquitous $5 \mathrm{G}$ networks, which may contain one sensor every $10 \mathrm{~m}$ for the same area or 10,000 sensors per $\mathrm{km}^{2}$ [11]. Our experimentation explores increasing device numbers from 1 to 1000 in increments of 100 for air quality sensors, and 1 to 50 in increments of 10 for the spectral cameras. We measure the latency and bandwidth of WiFi connections from devices to $5 \mathrm{G}$ smartphone, and the 5G/LTE connections from the smartphone to the edge server. Each thread transmits data to the $5 \mathrm{G}$ smartphone at an interval of $2 \mathrm{~s}$ for the air quality sensor data and a period of $0.05 \mathrm{~s}$ for the spectral image data.

Figures 4(a), 4(b), 4(f), and 4(g) compare the latency and throughput of $5 \mathrm{G}$ and LTE for data transfer from the $5 \mathrm{G}$ smartphone to the edge server, when the number of sensors and cameras are increased. We observe that, 5G provides significantly reduced latency over LTE. The throughputs are comparable for $5 \mathrm{G}$ and LTE connections in the scalability test, with the spectral data achieving a slightly larger difference than the sensor data. Transferring spectral camera data achieves a higher throughput with a much lower number of devices as the amount of transferred data is larger than that of the sensor.

We analyze proposed algorithms (EDNet and EnvNet) on the edge server and compare their performance against other alternative algorithms. Figures $4(\mathrm{c})$ and 4 (h) present the breakdown latency required to transfer data from the devices to the $5 \mathrm{G}$ smartphone with $\mathrm{WiFi}$, from the $5 \mathrm{G}$ phone to edge server with $5 \mathrm{G}$, and the model inference time on the edge server. 

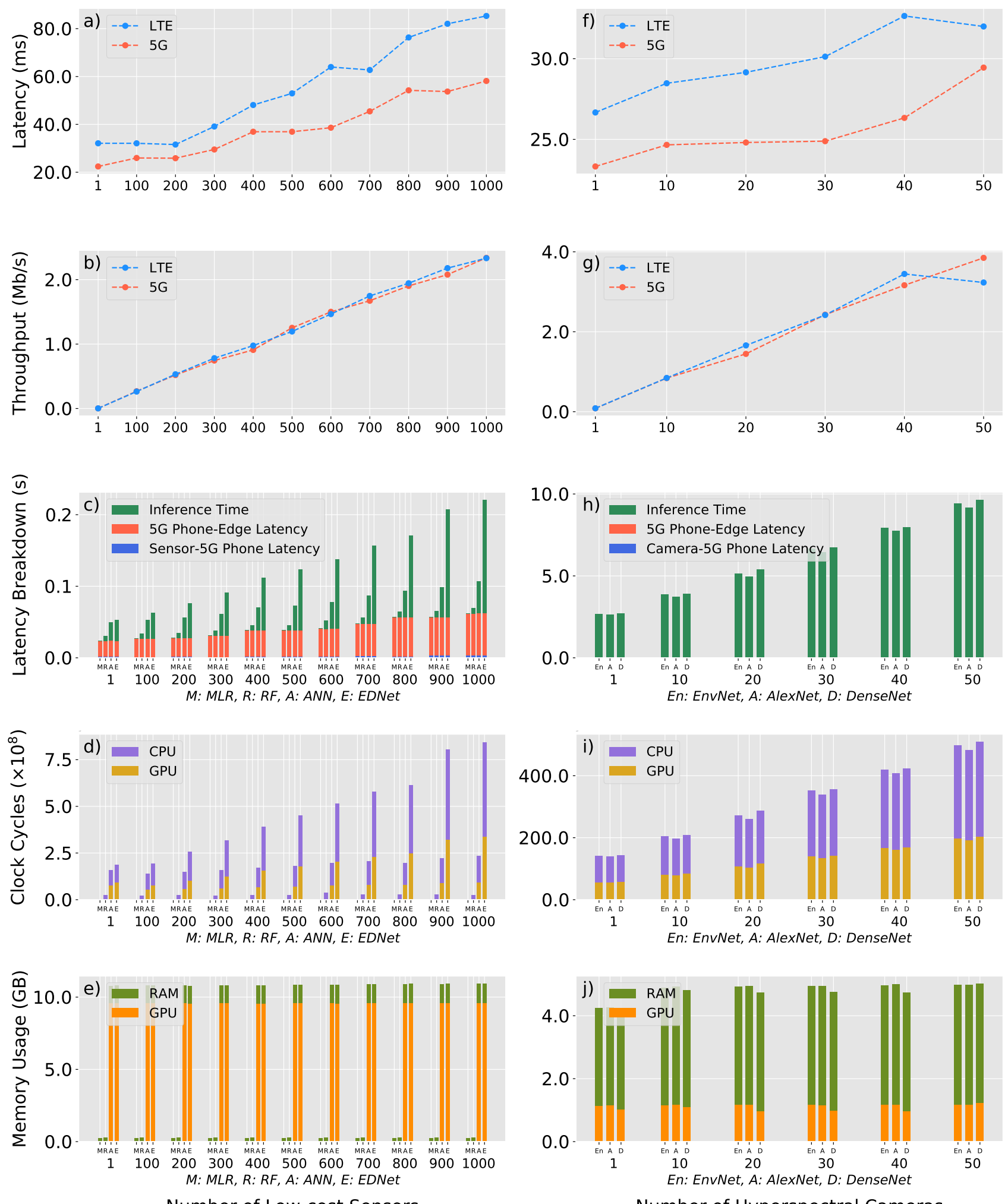

Fig. 4: Left: scalability results for low-cost air quality sensors: (a) Latency comparison of 5G and LTE, 5G provides a 28.0\% latency improvement over LTE; (b) Throughput comparison of 5G and LTE, with a 1.14\% percentage difference between both types of connections; (c) Latency breakdown for low-cost sensor data communication and analysis, communication latency dominates in end-to-end latency related to RF, MLR and ANN algorithms, and inference time dominates in EDNet; (d) CPU and GPU clock cycles for the analysis; (e) GPU memory and RAM usage for analysis; Right: scalability results for hyperspectral specim IQ cameras: (f) Latency comparison of 5G and LTE, 5G provides a $17.6 \%$ latency improvement over LTE; (g) Throughput comparison of 5G and LTE, with a 6.86\% percentage difference between both connections; (h) Latency breakdown for camera images data communication and analysis, inference time dominates for spectral camera image processing algorithms; (i) CPU and GPU clock cycles for the analysis; (j) GPU memory and RAM usage for analysis. The analytics for both sensors and cameras include analyses of different AI algorithms, their results are labeled according to the keys below each plot. 
For both air quality sensors and hyperspectral cameras, as device number increases, the inference time increases linearly. With the hyperspectral imaging processing, the inference times are significantly higher than the data transfer. For example, the inference time of EnvNet is on average 221 times larger than the total data transfer latency, this is also evident in AlexNet and DenseNet. Comparing the sensor calibration models inference times, their values do not dominate the data transfer latency, as with the hyperspectral analysis. For EDNet, the inference time is on average 2.04 times larger than the total data transfer latency. And EDNet in particular has a noticeable increase in inference time as the number of sensors increases, this is due to the model being more computationally complex than others.

We further investigate computation resource usage of the inference on the edge server. Figures $4(\mathrm{~d})$ and 4 (i) present estimations of the CPU and GPU clock cycles. We observe in both systems, the CPU utilizes more clock cycles for inference. However, the hyperspectral image processing utilizes more clock cycles than sensor calibration, as the CPU must transform the data from 204 to 29 channels before on-GPU analysis is performed. When comparing the memory usage, the inference with air quality sensors utilizes more GPU memory than it does RAM (Figure 4(e)), this is reversed with the hyperspectral image processing (Figure $4(\mathrm{j})$ ). Inter-comparisons between the different AI algorithms' performances in both clock cycles and memory usage metrics shows that: 1) EDNet and the ANN both require more resources than the MLR or RF algorithms. The implementations of both MLR and RF do not utilize the GPU, therefore display no GPU usage. 2) EDNet achieves the best accuracy compared to ANN, RF, and MLR, but requires more resources and inference time on the edge. Therefore, there is a trade-off to be considered according to available resources and system level requirements. 3) EnvNet, AlexNet, and DenseNet require comparable computation resources when analyzing the hyperspectral images across increasing camera numbers.

\section{CONCLUSiON}

$5 \mathrm{G}$ and AI have paved the way for the development of novel services and applications for massive-scale air quality monitoring, which reduces individuals' exposure to poor air quality. This paper addresses significant challenges pertaining to real-time air quality sensing and analysis by leveraging 5G edge, AI methods, and massive deployment of devices in urban environments. Our contributions are threefold: 1) we develop a scalable air quality monitoring architecture supported by $5 \mathrm{G}$ edge; 2) we deploy two AI systems, i.e., low-cost sensor calibration and hyperspectral image processing with alternative algorithms on 5GTN; and 3) we conduct a comprehensive scalability analytics of communication and computation resources on edge servers. Our experimentation verifies 1) $5 \mathrm{G}$ supports scalable deployment of air quality monitoring with low latency; and 2) different AI algorithms can be deployed on the 5G edge servers for latency-sensitive analysis.
In future research, we will explore run-time calibration of the hierarchical sensors as well as investigate approaches for processing real-time image and video data from hyperspectral cameras for air pollutant detection. We will further investigate how to leverage local knowledge at edge servers to design more complex algorithms by deploying collaborative calibration, transfer learning, and federated learning for sensor calibration and data analysis.

\section{ACKNOWLEDGMENT}

This work is partially funded by Academy of Finland, grant numbers 3196669, 319670, 325774, 326305, 325570, 324576 and 335934 . This work is partially supported by the Helsinki Center for Data Science (HiDATA) program, the European Union through the Urban Innovative Action Healthy Outdoor Premises for Everyone (UIA03-240), Business Finland Project 6884/31/2018 MegaSense Smart City, and project 16214817 from the Research Grants Council of Hong Kong. This work is also partially funded by the Ministry of Science and Technology of the People's Republic of China (2019YFC0214701), the National Natural Science Foundation of China (41877306) and Beijing University of Chemical Technology. The authors wish to acknowledge CSC-IT Center for Science, Finland, for computational resources.

\section{REFERENCES}

[1] J. M. Schleicher, M. Vögler, S. Dustdar, and C. Inzinger, "Enabling a smart city application ecosystem: requirements and architectural aspects," IEEE Internet Computing, vol. 20, no. 2, pp. 58-65, 2016.

[2] IHME, "Global Burden of Air Pollution," http://www.healthdata.org/ infographic/global-burden-air-pollution. April 2016, [Online; accessed 28-July-2020]

[3] N. H. Motlagh, E. Lagerspetz, P. Nurmi, X. Li, S. Varjonen, J. Mineraud, M. Siekkinen, A. Rebeiro-Hargrave, T. Hussein, T. Petaja, M. Kulmala, and S. Tarkoma, "Toward massive scale air quality monitoring," IEEE Communications Magazine, vol. 58, no. 2, pp. 54-59, February 2020.

[4] 5GTN, "5G Test Network," https://5gtn.fi/. 2019, [Online; accessed 28July-2020].

[5] 3GPP, "Feasibility study on new services and markets technology enablers," 3rd Generation Partnership Project, Tech. Rep., September 2016.

[6] M. Gusev and S. Dustdar, "Going back to the roots-the evolution of edge computing, an iot perspective," IEEE Internet Computing, vol. 22, no. 2, pp. 5-15, 2018.

[7] Y. Lin, W. Dong, and Y. Chen, "Calibrating low-cost sensors by a two-phase learning approach for urban air quality measurement," Proceedings of the ACM on Interactive, Mobile, Wearable and Ubiquitous Technologies, vol. 2, no. 1, pp. 1-18, 2018.

[8] J. Kuula, T. Mäkelä, M. Aurela, K. Teinilä, S. Varjonen, Ó. González, and H. Timonen, "Laboratory evaluation of particle-size selectivity of optical low-cost particulate matter sensors," Atmospheric Measurement Techniques, vol. 13, no. 5, pp. 2413-2423, 2020.

[9] M. Kulmala, "Build a global earth observatory," Nature, vol. 553, pp. 21-23, 012018

[10] F. Concas, J. Mineraud, E. Lagerspetz, S. Varjonen, X. Liu, K. Puolamäki, P. Nurmi, and S. Tarkoma, "Low-cost outdoor air quality monitoring and sensor calibration: A survey and critical analysis," $A C M$ transactions on sensor networks, 2021, arXiv:1912.06384 [eess.SP].

[11] Nokia, "The outlook for intelligent air quality monitoring in megacities as a 5G service," Nokia Corporation, Tech. Rep., 2018. [Online]. Available: https://onestore.nokia.com/asset/202208

[12] K. He, J. Sun, and X. Tang, "Single image haze removal using dark channel prior," IEEE transactions on pattern analysis and machine intelligence, vol. 33, no. 12, pp. 2341-2353, 2010. 
[13] A. Mehta, H. Sinha, P. Narang, and M. Mandal, "Hidegan: A hyperspectral-guided image dehazing gan," in Proceedings of the IEEE/CVF Conference on Computer Vision and Pattern Recognition Workshops, 2020, pp. 212-213.

[14] B. Li, X. Peng, Z. Wang, J. Xu, and D. Feng, "Aod-net: All-in-one dehazing network," in Proceedings of the IEEE International Conference on Computer Vision, 2017, pp. 4770-4778.

[15] J. Behmann, K. Acebron, D. Emin, S. Bennertz, S. Matsubara S. Thomas, D. Bohnenkamp, M. T. Kuska, J. Jussila, H. Salo et al., "Specim iq: evaluation of a new, miniaturized handheld hyperspectral camera and its application for plant phenotyping and disease detection," Sensors, vol. 18, no. 2, p. 441, 2018.

[16] K. He, X. Zhang, S. Ren, and J. Sun, "Deep residual learning for image recognition," in Proceedings of the IEEE conference on computer vision and pattern recognition, 2016, pp. 770-778.

[17] Institute for Atmospheric and Earth System Research (INAR), "SMEAR station network," https://www.atm.helsinki.fi/globalsmear/index.php/sta-

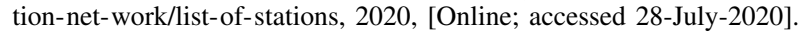

[18] A. Krizhevsky, I. Sutskever, and G. E. Hinton, "Imagenet classification with deep convolutional neural networks," Communications of the ACM, vol. 60, no. 6, pp. 84-90, 2017.

[19] G. Huang, Z. Liu, L. Van Der Maaten, and K. Q. Weinberger, "Densely connected convolutional networks," in Proceedings of the IEEE conference on computer vision and pattern recognition, 2017, pp. 4700-4708.

\section{BIOGRAPHIES}

Xiang Su: is an Academy of Finland postdoc fellow and a senior postdoctoral researcher in computer science at the University of Helsinki. He received his $\mathrm{PhD}$ in technology from the University of Oulu in 2016. Dr. Su has extensive expertise on the Internet of Things, edge computing, and augmented reality. Contact him at xiang.su@helsinki.fi.

Xiaoli Liu: is a postdoctoral researcher in computer science at the University of Helsinki. She received her $\mathrm{PhD}$ in mathematics and statistics at the University of Helsinki in 2017. Her research interests include big data analysis, AI, Internet of Things, and wearable computing. Contact her at xiaoli.liu@helsinki.fi.

Naser Hossein Motlagh: is a postdoctoral researcher in computer science at the University of Helsinki. He completed his D.Sc. in networking technology from Aalto University in 2018. His research interests include Internet of Things, WSN, UAVs and AUVs. Contact him at naser.motlagh@helsinki.fi.

Jacky Cao: is a $\mathrm{PhD}$ student at the University of Oulu. He received his MPhys. Physics degree from Durham University in 2019. His research interests include mobile augmented reality, edge computing, and $5 \mathrm{G}$ networks. Contact him at jacky.cao@oulu.fi.

Peifeng Su: is a PhD student at the department of Geosciences and Geography, University of Helsinki. His research interests include remote sensing, image processing, and deep learning. Contact him at peifeng.su@helsinki.fi.

Petri Pellikka: is a full professor at the Department of Geosciences and Geography, University of Helsinki. His research interests include remote sensing, earth observation and environmental sensing and applications of those in land cover, land use and climate change research. Contact him at petri.pellikka@helsinki.fi.

Yongchun Liu: is a full professor at Beijing University of Chemical Technology. He received his $\mathrm{PhD}$ from Chinese Academy of Sciences in 2008. His research interests focus on atmospheric chemistry and its environmental impacts, such as the heterogeneous reaction mechanism and kinetics of trace gases on ambient relevant particle matter, chemical aging and toxicological evolution of carbonaceous aerosols, and source apportionment of air pollutants. Contact him at liuyc@buct.edu.cn.

Tuukka Petäjä: is a full professor at the Institute for Atmospheric and Earth System Research, University of Helsinki. He completed his PhD in Physics at the University of Helsinki in 2006. His research interests include atmospheric aerosol particles and their role in climate change and air quality. Contact him at tuukka.petaja@helsinki.fi

Markku Kulmala: is an Academy Professor and head of the Institute for Atmospheric and Earth System Research, University of Helsinki. He completed his $\mathrm{PhD}$ in Theoretical Physics at the University of Helsinki in 1988. His research interests include nanoparticle formation in the atmosphere, biosphere-atmosphere interactions and feedbacks. Contact him at markku.kulmala@helsinki.fi.

Pan Hui: is the Nokia Chair Professor in Data Science and Professor of Computer Science at the University of Helsinki. He is also the director of the HKUST-DT System and Media Lab at the Hong Kong University of Science and Technology. He received his $\mathrm{PhD}$ from the Computer Laboratory at University of Cambridge. $\mathrm{He}$ is an IEEE Fellow, an ACM Distinguished Scientist, and a member of Academia Europaea. Contact him at pan.hui@helsinki.fi.

Sasu Tarkoma: is a full professor at the Department of Computer Science, University of Helsinki. He completed his $\mathrm{PhD}$ in Computer Science at the University of Helsinki in 2006. His research interests include mobile computing, Internet technologies, and AI. Contact him at sasu.tarkoma@helsinki.fi. 